\title{
1
}

\section{Sexuality and the Mental Health Professional}

Study in the field of sexology (the study of human sexuality) calls for sexpositivity, the outlook "that all consensual expressions of sexuality are valid" (Kimmes et al., 2015, p. 289). Using a sex-positive framework to understand sexuality, it is the person's own experience and meaning-making that are important, rather than social norms regarding sexual behavior (Coleman et al., 2018). In this regard, much of what is called "sexual dysfunction" is a result of incongruence between an individual's internal experience and their external environment, which is replete with sex-negative beliefs that fail to support sexual health and pleasure. It is the inherent value of a sex-positive world that created the impetus to write this textbook.

When I decided to become certified in sex therapy, my then supervisor, Stephen Braveman, early on stated something that stuck with me, "In our culture, no one escapes having some form of sexual damage." Unfortunately, this statement describes not only the influence of culture on lay people but also the mental health of healthcare professionals who treat them. (Note that the umbrella term providers will be used frequently throughout the text to be inclusive of everyone who treats sexual issues.) Consider how other systems, such as family, school, or peers, influenced your development of your sexual identity. Perhaps you experienced being sexually harassed on the schoolyard or the workplace. You may be the one in four women or the one in six men who were molested as children and who did not feel supported enough by parents or school to reveal it. You may have engaged in sex when you didn't want to-or even coerced someone into having sex with you-which affected your cognitive framework and approach to sexual engagement, even in safe relationships. Or perhaps more recently, you were educated as a provider in a setting where sexuality was a marginalized topic of study-an all-toocommon experience. 
Despite these and similar challenges, you hopefully grew up to be a sexually functioning adult capable of emotional and physical intimacy with a partner of your choosing, but there is a strong likelihood that you have had ongoing struggles with your sexuality, as do surprising numbers of men and women in the population as identified by Laumann et al. (1999) in the largest study of its kind. It is in regard to sexuality that providers are perhaps most like their clients, kept in the dark about its nature and discouraged from opening the door to understanding. Insight and knowledge into sexuality requires that providers pay attention to their own development, but our training reflects the reluctance of our culture as a whole to "go there" and attend to this aspect of the self of the provider, which is perhaps one of the most important tools providers have for treating clients. For most providers, learning how to address sexual topics requires specialized training that may be difficult to access, requiring travel and other expense.

What happens to our clients if we, ourselves, don't have or make the opportunity to understand our own sexuality? Can we call ourselves competent if we don't assess or treat the entire spectrum of human experience, including those that may be baffling or taboo? As self-appointed healers of mental health, I believe we must take responsibility to heal the whole person, including the sexual part of the self. Otherwise, we may inadvertently sustain or contribute to a client's struggle with sexuality. Providers have yet to fully recognize that sexual problems can contribute to depression, anxiety, trauma, eating disorders, substance abuse, pain disorders, and so on; conversely, such problems can affect a person's sexuality, yet remain unaddressed in the provider's office. Yet if we don't "do sex" as part of our practice, we may miss out on an essential part of what is troubling our clients (Zeglin, 2016).

Consider Len, a client in his mid-60s who was so guilt-ridden after his first attempt at intercourse at age 20 that he struggled with erectile dysfunction for the 40-plus years that followed. Len became afflicted with depression and sought relief through therapy, medications, and, at times, alcohol. In all his time spent in counseling, no provider had asked him about his sex lifeuntil he mentioned a urology appointment to a provider he was seeing at the time. Concerned he might be ill, the provider asked more about the appointment. The question prompted Len to cry as he told the provider about his "impotence." Fortunately, the provider realized that this was a long-standing, complex issue for Len and a referral was made for my help. After months of treatment Len and his partner were, at last, able to experience sexual pleasure as they had always desired.

But how different might Len's life have been if someone had earlier inquired, "How's your sex life?" Len's treatment was deterred by a culture in which nonspecialized providers lack training or comfort discussing sexual topics (Burnes et al., 2017). He needed a provider who could use a systemic approach to understand why his struggle was so long-standing and intense, which had to do with his development in an extremely restrictive family, church, and community environment. The restrictive environment caused Len undue shame about his sexual needs, which in turn caused him to lose his erection in his first sexual encounter. Later in life, changes in Len's biological system made attaining any kind of erection almost impossible, adding 
further frustration. Only when Len's frustration became more painful than his secret could, he broke out of his shell and reached out for help.

Clients come to providers to help them solve all kinds of problems, which may include sexual concerns. The Laumann survey (Laumann et al., 1999) of male and female sexual problems-the largest of its kind-reported that about $60 \%$ of women and $30 \%$ of men have had some type of sexual dysfunction. With numbers like these, it is safe to assume that a good portion of your current clients have a sexual problem. If you do not ask, however, you may never hear about these issues. But clients sometimes do tell me, an identified sex therapist, some of the reasons why they left a former provider who didn't talk about sex:

- One woman found the courage to seek help for the fact that she was still a virgin 7 years after her wedding night due to vaginismus (spasm of the vagina that prevents penetration), but she could not bring herself to tell the therapist about her problem. Therapy focused on an incident of molestation as a child, but the therapist never asked about sex. The client got the message loud and clear: Sex is not spoken here.

- A couple trying to get past an affair that nearly destroyed their marriage couldn't bring themselves to tell their "uptight" therapist that they had enjoyed swinging early in their relationship. The couple's history added to their confusion about boundaries and rules in the marriage, but their weeks in therapy were useless because they feared the therapist would judge them if they disclosed their early history.

- Amal was failing his undergraduate courses and was referred to a specialist in cognitive behavioral therapy, where treatment began for generalized anxiety. Behind the symptoms, however, was a sexual secret: Amal was contacting male strangers for sex, drive to meet up, ring the doorbell, and then run away with his heart racing before the door opened. He sought my help, and I encouraged him to open up to his other therapist, but due to his Middle Eastern background and fears around homosexuality, it took weeks to do so. He later expressed to me, however, that he should not need therapy to talk to a therapist about sex and wished that the other therapist had asked.

No one, least of all our clients, wants to be judged as defective, naive, or perverse. People want reassurance that they are acceptable or lovable even when their sexuality is outside the norm, which is a heterosexual person engaging in penis-vagina intercourse. People who engage in bondage, who enjoy watching sexually explicit material, or partners who love one another but who grow tired of monogamy and want to explore possibilities often do so in fear of being judged by others.

The fact that providers don't talk about sex may be through no fault of their own. If you are reading this textbook because you are enrolled in a full graduate course in sex therapy, you are in the minority. There are programs that offer zero hours of education in sex therapy or at minimum one credit-10 to 12 hours - of education. In a survey of 110 Canadian and American psychologists, Miller and Byers (2010) identified that psychologists lacked the ability to 
directly address clients' sexual concerns ("Skills Self-Efficacy") and to relate accurate information about sex ("Information Self-Efficacy"). In another survey of 188 Canadian psychologists, $60 \%$ did not seem to have the training or comfort level to do so (Reissing \& Giulio, 2010). Graduate programs often do not have faculty trained in sexual issues or they marginalize sexual health topics (Zamboni \& Zaid, 2017). Physicians and nurses who ostensibly know the most about sexual anatomy and hormones fare no better; for example, when faced with oncology patients whom they know to have sexual issues but do not have the knowledge or comfort level to help them (Ussher et al., 2013).

Of course, some providers do ask about sex. In another survey, about half of therapists reported that they "always" ask about sex (Miller \& Byers, 2009). But people in general (including therapists) overreport their comfort level with sexuality to appear normal or "cool." What about the other half of therapists? There is an adage that we are always communicating, even if we say nothing. Therapists' silence about sex in the therapy room sends many messages, but perhaps the loudest is please don't talk about sex!

\section{WHY THE SILENCE ABOUT SEX?}

Almost everyone is raised in an atmosphere of secrecy when it comes to the emotional and psychological aspects of sex. The reasons are complex. Depending on one's religious perspective, a person's very conception can be shrouded with mystery. As we grow, we covertly observe romantic and sexual behaviors between adults of which we are told little. We experience our own biological urges, such as a desire to masturbate, but are given neither tacit nor explicit permission to ask questions. If we begin to identify in a way that we don't see portrayed in the media, perhaps as a sexual or gender minority, then we assume that there is something wrong with us. If we ask about sex, we may not get an answer-or we may get a lecture on why a particular sexual topic is naughty or dirty, something good girls and boys avoid. Although many of today's parents talk openly with their children about sex, there are still households where sex is a forbidden topic. Only 11 of 50 states have a comprehensive sex education program that includes information on healthy sexual relationships or the issue of sexual assault (Shapiro \& Brown, 2018).

Our physical and psychological sexual development takes place on different planes, and interactions between and among systems reflect this. Secondary sexual characteristics signal to adults around us that we must be protected from such risks as unwanted pregnancy or disease. Meanwhile, we are left to privately grapple with sexual dreams and fantasies, curiosity, and desire. Reflecting what is still often a puritanical culture, we aren't given information about giving and receiving sexual pleasure. The complexities of a sexual relationship are explained away with myths like, "All men are interested in is sex" or "Once you get married, your sex life will disappear."

We certainly aren't told how to manage feelings of sexual inadequacy, so if a problem comes up like difficulty having an orgasm or getting an erection, we may keep it under wraps for decades. As a society, we also may act as if sexual problems appear suddenly in adulthood. However, as I have learned 
from my clients, teens (who on average in the United States begin having intercourse at around age 15) have sexual problems that plague them, sometimes leading to depression, eating disorders, alcoholism, and other mental illness. As providers know all too well, secrecy and silence are associated with feelings of shame and guilt that can contribute to overall poor mental health. Nowhere, perhaps, is this truer than when it affects our sexual development.

It doesn't help that sex does have a murky side. Subjects such as sexual abuse, assault, and rape tend to be hushed by the victim and, if they learn about it, family members or friends. If the media gets hold of such a story, it is sensationalized, which may cause victims to further retreat away from possible unwanted attention. Meanwhile, media attention such as that placed on the 82,000 lawsuits alleging sexual abuse filed against the Boy Scouts of America do little to address underlying problems, such as the need to educate children at an early age about appropriate physical boundaries, the right to say no to unwanted touch, and permission to tell if someone harms them (Baker, 2020). Our culture also has done a poor job of acknowledging that pedophiles and people with other difficult sex and social problems do exist and frequently deserve compassionate treatment (Cantor, 2012).

For teens and young adults, date rape is distressingly common on college campuses; many young women have shared with me that the reputed response of campus security was so lukewarm that they made a decision not to report. Yet its effects can linger well into adulthood. Today's young adults are also the first to grow up in the digital age. Many have spent so much time viewing internet pornography that they neglected to develop the social and sexual skills to have a real partner. How might things be different if they were raised in a culture that acknowledged sexual needs, made it easy to attain contraception, and talked openly about what it means to be in a healthy sexual relationship?

Providers are not immune to such sex negativity. Pope and FeldmanSummers (1992) report that about two-thirds of female and one-third of male therapists responding to a survey regarding sexual abuse among therapists had experienced molestation, the majority of incidents associated with a close relative. It isn't difficult to surmise that one reason therapists may avoid the topic of sex is that they have been victims of sexual abuse or exposed to other types of negative sexual experiences, from mild harassment to outright assault. Although therapists may be drawn to the field to help other victims become survivors, they may be disappointed with how little attention their graduate training gives to the sexual late effects of abuse.

The negative effects of such experiences may intensify what Saakvitne and Pearlman (1996) called vicarious traumatization. Vicarious traumatization is said to occur when a provider empathizes with and feels responsibility for a client's pain and suffering. Listening to sexual material can be difficult for providers if they have their own sexual struggles. Like our clients, we may have trouble setting appropriate sexual boundaries, struggle with questions about the morality of sexual practices (anything from casual "hook-ups" to looking at-or even engaging in the making of-hardcore pornography), be dealing with our own sexual inhibitions, be worrying about a partner's sexual function after cancer or other illness, or simply have basic concerns about our 
own physical appearance and sexual attractiveness. Discussing such matters may create such distress for the provider that they are simply avoided.

Another reason for many providers' silence about sex is the fear of becoming isolated or being ostracized by colleagues. Not only do providers fear being judged by clients for expressing interest in their sex life but also by other providers who find dealing with sexual problems too uncomfortable or distasteful. For example, I recently lucked upon an opportunity to share space in a pretty office with a psychoanalyst. My deposit was promptly returned because, on reflection, the analyst decided that my sex therapy practice was not a good fit for her office, presumably since she and her clients might happen upon my (drooling?) clients in the waiting room. Working with sexual problems can also feel unsafe in certain communities because of the social and political climate. In California, condoms and lubricants are sold over the counter at the drugstore, but there are still parts of the country where such items are difficult to procure. Designating oneself as a sex therapist in such areas can mean opening one's self up to ridicule or harassment.

Providers may also be quiet about sex due to strict training in laws and ethics concerning sexual contact with clients, a topic covered in detail in Chapter 19. Providers are rightly warned that because there is an inherent power differential in the relationship with the client-who is dependent on us for emotional support-the potential for doing serious emotional damage by acting out sexual urges comes with enormous risk. Little is said, however, about reconciling the need to manage one's sexual attraction or arousal while discussing sex in a therapeutic manner with the client. In any case, stern warnings about curbing one's sexual feelings may send the message that it's best not to deal with sex in the treatment room.

A final reason for providers' reticence about sex concerns diversity. While learning how to provide ethical treatment to people from multiple cultures has been a priority in many training programs, working with sexually diverse people in a sex-positive way has not. If providers are to properly address client sexuality, then they must not only have appropriate training regarding sexual function but also recognize their implicit heteronormative bias and focus on sex for procreation rather than recreation. In order to do so, providers need to develop their capacity for self-reflection, client affirmation, and normalizing of sexual behaviors (Berry \& Lezos, 2017). Providers, however, are not solely to blame. In a content analysis of marriage and family therapy and family studies journals, of 13,919 articles published within 15 journals, just 137 focused on sexuality or sex therapy (Jones et al., 2019). If academia lacks attention to this area of study, then there are implications for graduate training programs.

On the other hand, perhaps none of these concerns apply to you. You might be someone who can't wait to talk to clients about sex. Maybe you are a graduate student who wants to write a dissertation on a sexually related topic or to work at an agency that isn't afraid that you will ask clients about their sex lives. If so, be fearless. Give your dissertation or agency chair a copy of the studies cited about the need for providers who can work with clients regarding sex. Show them this book. Offer to get outside consultation or supervision from a practicing and/or certified sex therapist. Find professors and others 
in your community who will support you. Just be persistent and even pesky, because your community needs someone like you.

For the rest, have the courage to talk about sex! Consider that you are about to become a step ahead of most providers as you learn more about sexuality. You won't be a provider who "doesn't 'do' sex." You will be able to help clients overcome their deepest fears so that they can enjoy one of life's pleasures without undue shame or guilt. Increasing a client's capacity to love and be loved often has a ripple effect in the client's life, giving them the optimism, confidence, and freedom to tackle other developmental milestones such as finding a healthy relationship or even making a much-feared job change. Our sexuality is such a core part of who we are, and as providers we are fortunate to be in a position to help our clients achieve health in this critical area of human existence.

An important note before you delve into the rest of the book: It is impossible to cover all the nuances of human sexuality in a single volume. Please take advantage of the Resources at the end of each chapter if there is a topic area in which you want to expand or deepen your knowledge. Also, you may find that you disagree with some of what is written in these pages, as sexology is a value-laden area of study. When this occurs, it can be a good time to examine your own upbringing and beliefs about what it means to be a sexual being. You may identify assumptions that will turn your thinking on its head or you may find that you are comfortable with who you are. Either way, sexology is an endlessly fascinating topic that I hope you will find an enjoyable one to study.

\section{TAKE-AWAY POINTS}

1. Sexual health is part of good mental health and should not be marginalized as a topic of study.

2. Sexual problems can be the cause as well as the result of mental illness.

3. Providers may not talk about sex for the same reasons as clients, for example, shame and embarrassment regarding the topic. Providers may also have faced sexual trauma or experienced vicarious traumatization.

4. Providers are sometimes told to avoid addressing sexual issues due to the fear that they may violate their ethics. They may need to get in touch with their courageous side to talk about sex.

5. Providers need to have a thorough understanding of issues faced by sexual- and gender-minority identified clients in order to provide ethical treatment to everyone seeking services.

6. Providers can become more comfortable with sexuality by seeking sex-positive instructors, reading more about sexuality, and seeking appropriate supervision with clients.

\section{ACTIVITIES}

1. Whether you have refrained from talking about sex with your clients or do so on a regular basis, it can be critical to examine your own attitudes about human sexuality. In a "sexological journal," write about influences you have experienced in your sexual development. 
2. What steps have you taken, or will you take to increase your comfort in talking with clients about sex? List them in your journal.

3. Locate a colleague with whom you can consult or from whom you can receive formal supervision of cases that present with sexual issues. Keep notes of your consultations in your journal.

4. Either for yourself or with your classmates, keep a running list of academic and popular podcasts, websites, and other resources about sexuality.

\section{RESOURCES}

The American Psychological Association has a list of resources for professionals regarding ethical treatment of LGBTQ+ identified people at www.apa.org/pi/lgbt/resources /practitioner

The Guttmacher Institute publishes well-researched reports on topics of sexual health and reproduction. www.guttmacher.org/\#

Joannides, P. (2017). The guide to getting it on. Goofy Foot Press. Informational book about sexual topics of all sorts for the curious.

The Kinsey Institute website has produced a page of resources for professionals interested in the field of sexology entitled Kinsey Confidential. Kinsey Confidential can be found at www.kinseyconfidential.org/resources/sex-research-sex-therapy

The National Coalition for Sexual Health (NCSH) also has resources for professionals as well as the public. The site also keeps a running list of current news articles, "Sexual Health in the News." NCSH can be found at www.nationalcoalitionforsexualhealth .org/

Scarleteen is a longstanding resource for information about sexuality geared for teens, but it is excellent for adults, too. www.scarleteen.com/

\section{KEY REFERENCES}

Only key references appear in the print edition. The full reference list appears in the digital product found on http://connect.springerpub.com/content/book/978-0-8261-3589-6 /part/sec01/chapter/ch01

Berry, M. D., \& Lezos, A. N. (2017). Inclusive sex therapy practices: A qualitative study of the techniques sex therapists use when working with diverse sexual populations. Sexual and Relationship Therapy, 32(1), 2-21.

Burnes, T. R., Singh, A. A., \& Witherspoon, R. G. (2017). Sex positivity and counseling psychology: An introduction to the major contribution. The Counseling Psychologist, 45(4), 470-486.

Coleman, E., Dickenson, J. A., Girard, A., Rider, G. N., Candelario-Pérez, L. E., BeckerWarner, R., Kovic, A. G., \& Munns, R. (2018). An integrative biopsychosocial and sex positive model of understanding and treatment of impulsive/compulsive sexual behavior. Sexual Addiction \& Compulsivity, 25(2-3), 125-152.

Shapiro, S., \& Brown, C. (2018, May 9). Sex education standards across the states. Center for American Progress. www.americanprogress.org/issues/education-k-12 /reports/2018/05/09/450158/sex-education-standards-across-states /

Zamboni, B. D., \& Zaid, S. J. (2017). Human sexuality education in marriage and family therapy graduate programs. Journal of Marital and Family Therapy, 43(4), 605-616. 


\section{REFERENCES}

Baker, M. (2020, November 15). Sex-abuse claims against Boy Scouts now surpasses 82,000. The New York Times. www.nytimes.com/2020/11/15/us/boy-scouts-abuse -claims-bankruptcy.html

Cantor, C. (2012). Do pedophiles deserve sympathy? www.cnn.com/2012/06/21/opinion /cantor-pedophila-sandusky

Jones, A. C., Johnson, N. C., Wenglein, S., \& Elshershaby, S. T. (2019). The state of sex research in MFT and family studies literature: A seventeen-year content analysis. Journal of Marital and Family Therapy, 45(2), 275-295.

Kimmes, J. G., Mallory, A. B., Cameron, C., \& Köse, Ö. (2015). A treatment model for anxietyrelated sexual dysfunctions using mindfulness meditation within a sex-positive framework. Sexual \& Relationship Therapy, 30(2), 286-296. 10.1080/14681994.2015.1013023

Laumann, E. O., Paik, A., \& Rosen, R. C. (1999). The epidemiology of erectile dysfunction: Results from the National Health and Social Life Survey. International Journal of Impotence Research, 11, S60.

Miller, S. A., \& Byers, E. S. (2009). Psychologists' continuing education and training in sexuality. Journal of Sex \& Marital Therapy, 35(3), 206-219.

Miller, S. A., \& Byers, E. S. (2010). Psychologists' sexual education and training in graduate school. Canadian Journal of Behavioural Science [Revue canadienne des sciences du comportement], 42(2), 93.

Pope, K. S., \& Feldman-Summers, S. (1992). National survey of psychologists' sexual and physical abuse history and their evaluation of training and competence in these areas. Professional Psychology: Research and Practice, 23(5), 353-361.

Reissing, E. D., \& Giulio, G. D. (2010). Practicing clinical psychologists' provision of sexual health care services. Professional Psychology: Research and Practice, 41(1), 57.

Saakvitne, K. W., \& Pearlman, L. A. (1996). Transforming the pain: A workbook on vicarious traumatization. W. W. Norton.

Ussher, J. M., Perz, J., Gilbert, E., Wong, W. T., Mason, C., Hobbs, K., \& Kirsten, L. (2013). Talking about sex after cancer: A discourse analytic study of health care professional accounts of sexual communication with patients. Psychology and Health, 28(12), 1370-1390.

Zeglin, R. J. (2016). Sexual disorders in the DSM-5: Implications for counselors. Journal of Professional Counseling, Practice, Theory, and Research, 43(1), 17-31. 